\title{
DRITTE AUFLAGE.
}

\section{LEHRBUCH}

DER

\section{PRAKTISCHEN MEDICIN}

MIT BESONDERER RÜCKSICHT'

ALF

PATHOLOGISCHE ANATOMIE UND HISTOLOGIE

$\operatorname{los}$

Dв. C. F. KUNZE,

PRAKT ARZT IN HALLE A/S.

DRITTE MEHRFACH VERÄNDERTE UND VERMEHRTE AUFLAGE.

ZWEITER BAND.

LEIPZI G

VERLAG VON VEIT \& COMP.

1878. 



\section{LEHRBUCH}

\section{DER \\ PRAKTISCHEN MEDICIN \\ voN}

D. C. F. KUNZE.

ZWEITER BAND. 
Wir halten zum Abonnement empfohlen:

\title{
Deutsche Zeitschrift für praktische Medicin.
}

\author{
Begründet von Dr. C. F. Kunze.
}

Herausgegeben von Dr. B. Fränkel in Berlin.

Wöchentlich eine Nummer von $1-1 \frac{1}{2}$ Bogen Text in gross Quart.

Preis des Quartals 6 Mark.

Die „Deutsche Zeitschrift für praktische Medicin“، verfolgt den Zweck, den praktischen Arzt mit den neuen Erfahrungen und Leistungen der Medicin bekannt zu machen. Sie bringt in jeder Nummer mindestens einen Original-Artikel, der sich über eine Frage der Zeit in eingehender Weise verbreitet, ausführliche Referate über sämmtliche bedeutende Arbeiten des Inund Auslandes und zwar aus allen Gebieten der Medicin (pathologische Anatomie, innere Medicin, Chirurgie, Gynäkologie, Pädiatrik, Ophthalmologie, Physiologie, Anatomie u. s. w.), berichtet über die Verhandlungeu und Vorträge in den ärztlichen Vereinen, bespricht die wichtigsten Novitäten in unparteiischer Weise, veröffentlicht seltene oder durch sonstige Eigenthümlichkeiten ausgezeichnete Fälle, und giebt in den Abschnitten: Standesangelegenheiten, Tagesgeschichte und Personalien das Hierhergehörige der neuesten Zeit.

Die ,Deutsche Zeitschrift für praktische Medicin“ hat sich während der verhältnissmässig kurzen Zeit ihres Bestehens zahlreiche Freunde erworben und wird von Vielen als die die Bedürfnisse des praktischen Arztes am Besten deckende Zeitschrift betrachtet.

Probenummern liefert jede Buchhandlung unentgeltlich.

Zi beziehen durch alle Buchhandlungen und Postanstalten.

Die Verlagsbuchleandlung: Veit d Comp. in Leipsig. 


\title{
LEHRBUCH
}

DER

\section{PRAKTISCHEN MEDICIN}

MIT BESONDERER RÜCKSICHT

AUF

PATHOLOGISCHE ANATOMIE UND HISTOLOGE

voN

\author{
Dr. C. F. KUNZE, \\ PRAKT. ARZT IN HALLE $\mathrm{A} / \mathrm{s}$.
}

DRITTE MEHRFACH VERÄNDERTE UND VERMEHRTE AUFLAGE.

ZWEITER BAND.

LEIPZIG

VERLAG VON VEIT \& COMP.

1878. 
Das Recht der Herausgabe von Uebersetzungen in moderne Spracben vorbehalten.

Druck von Hundertstund \& Pries in Leipzig. 